\title{
System Analysis of a Hospital Information System Using UML Yi Tan ${ }^{1}$, Li Dong ${ }^{1}$, Yan $\mathrm{CaO}^{2}$, Lina Yang ${ }^{3}$, Hong Cao ${ }^{1, *}$
}

${ }^{1}$ Shandong Medical College, Jinan, Shandong, 250002, China

\author{
${ }^{2}$ Joint National and Local Engineering Research Center, Xi'an Technological University, Jinhua \\ North Road 4, Xi'an, Shaanxi, 710032, China \\ ${ }^{3}$ College of Humanities and Foreign Languages, Xi'an University of Science and Technology, \\ Yanta Road 58, Xi'an, Shaanxi, 710054, China \\ ”chsdmc@sina.com (corresponding author)
}

Keywords: HIS, System analysis, Information system, UML.

\begin{abstract}
According to patient-centered design principle, a Hospital Information System (HIS) can digitalize the whole daily process and information interaction of a hospital. The HIS requirements are analyzed and its use case diagrams and activity diagrams are drawn. According to the operational flow of departments, the HIS is composed of several functional sub-systems, including out-patient sub-system, medicine storehouse management sub-system, pharmacy management subsystem, electronic patient record management sub-system, leechdom dictionary management subsystem, user administration sub-system and login sub-system. The operation flows of various departments are recombined and their core flows and data flows are analyzed. The discussion in the paper defines the system requirements and architecture for further system realization.
\end{abstract}

\section{Introduction}

The development of hospital informationization goes through three phases, i.e., Hospital Information System (HIS) phase [1], Clinic Information System (CIS) phase and Globe Medical Information Service (GMIS) phase. At present, the hospital informationization in China is at the HIS phase. HIS construction is of great strategic importance for medical and health project modernization in China [2].

According to patient-centered design principle, the HIS can digitalize the whole daily process and information interaction of a hospital, including login, registration, charging, patient record, prescription, application sheet, drug dispensing, pharmacy management, daily work of medical treatment departments, etc. It effectively eliminates information transmission on paper. The HIS can establish an transparent, comfortable and fast medical treatment environment, providing patientoritented service and daily management information query service. Thus, it can improve the managing level of internal links, such as, leechdom, medical treatment, charging and so on in the hospital.. At the same time, the digitalized management, recording, storage, access and query of prescription, leechdom, and drug information are realized.

\section{System analysis and its UML diagrams}

\section{Use case diagrams}

After the HIS requirements are analyzed [3-5], its use case diagrams HIS are drawn. For example, the use case diagrams of out-patient charging, leechdom dictionary and user adminidtration are shown in Fig.1, Fig.2 and Fig.3 respectively. 


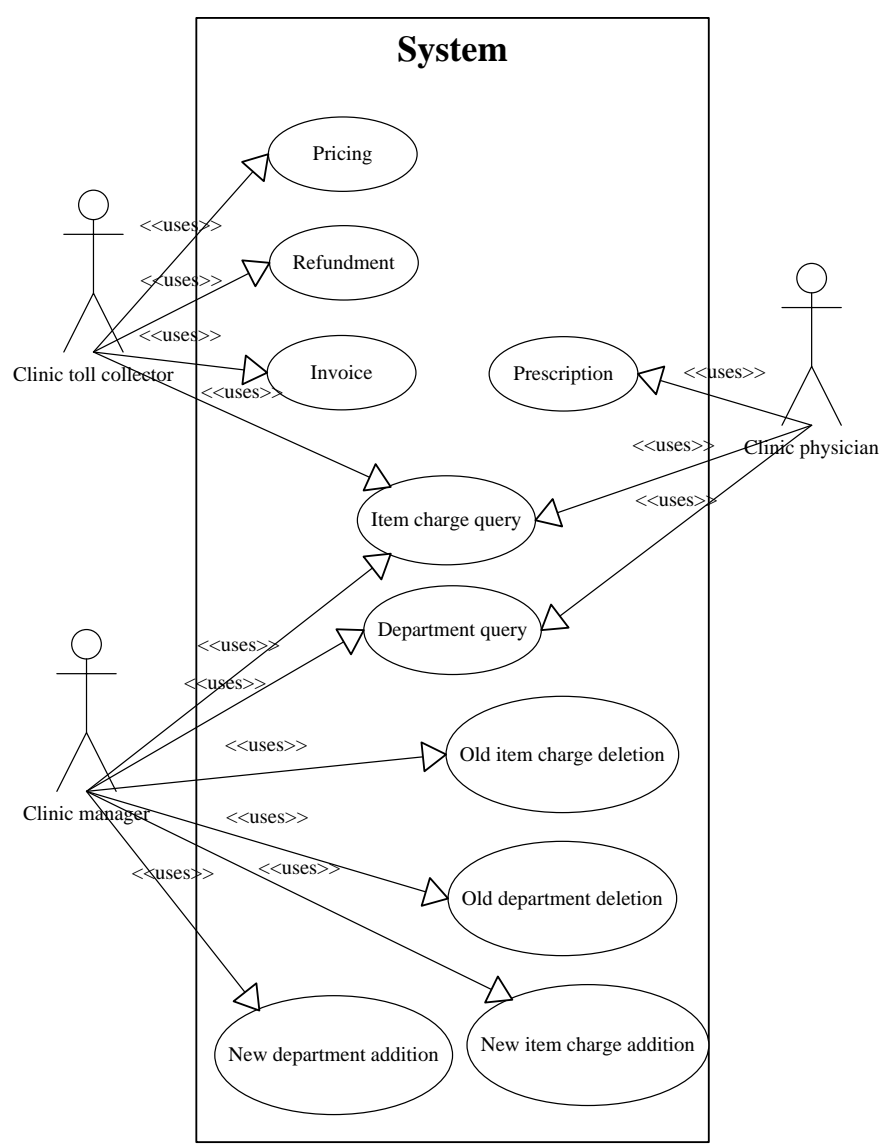

Figure 1 Use case diagram of out-patient charging

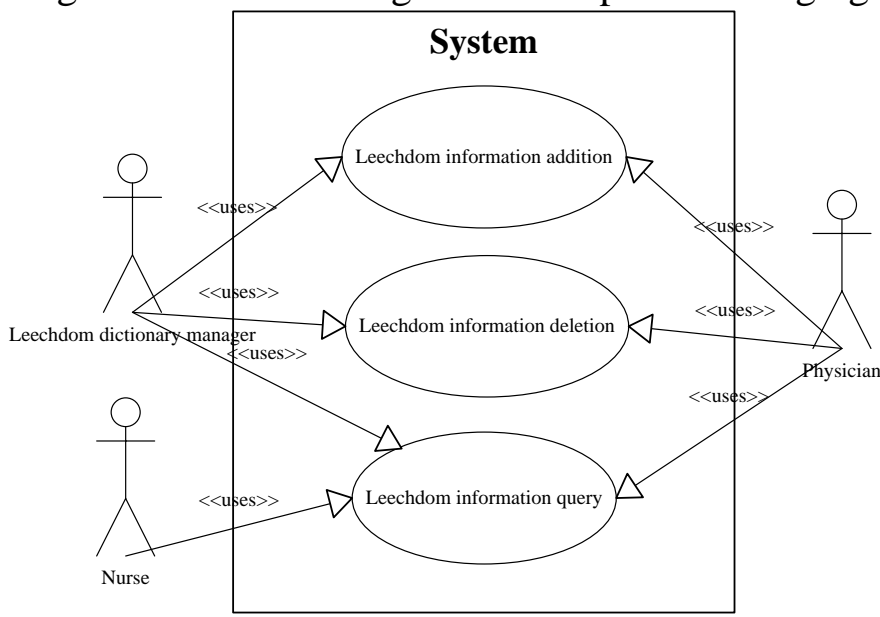

Figure 2 Use case disgram of leechdom dictionary

The maintenance and managing personnel are responsible for leechdom information addition and deletion. Physicians are also permitted to add or delete leechdom information. However, nurses are only permitted to query leechdom information. The use case diagram of user administration is shown in Fig.3. 


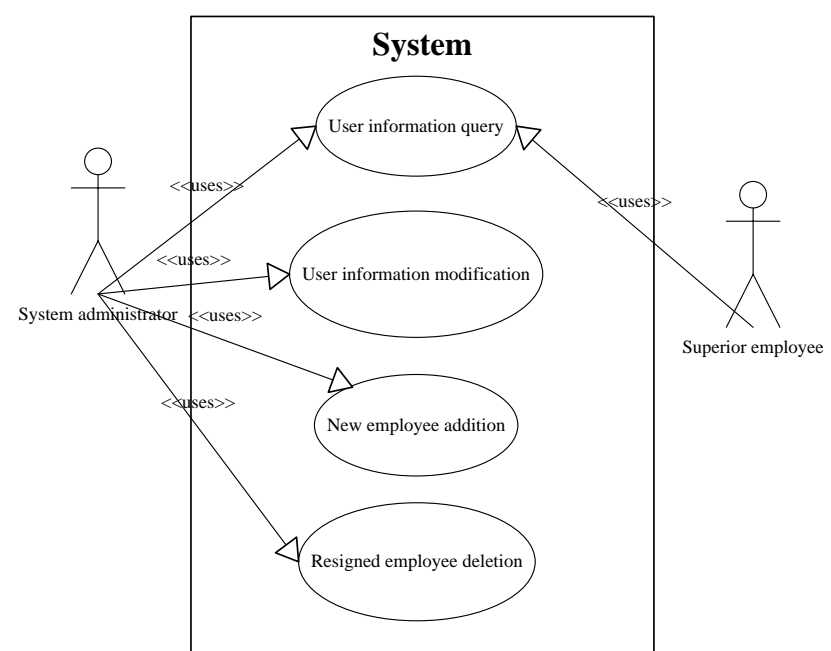

\section{Activity diagrams}

Figure 3 Use case diagram of user administration

The activities of each departments interact with each other. Inorder to make the relations among departments clear, the activity diagrams of the HIS are drwn. An activity diagram of HIS is shown in Fig.4 where out-patient department administrator, leechdom dictionary inquirer and system administrator control their own activities. For example, a leechdon inquirer chooses the needed leechdom information. Then, the system searches and displays the drugs list that meets the searching conditions. After the inquirer selects a drug from the list, the system dislplays the detailed drug information and permits the inquirer to modify or delete the drug.

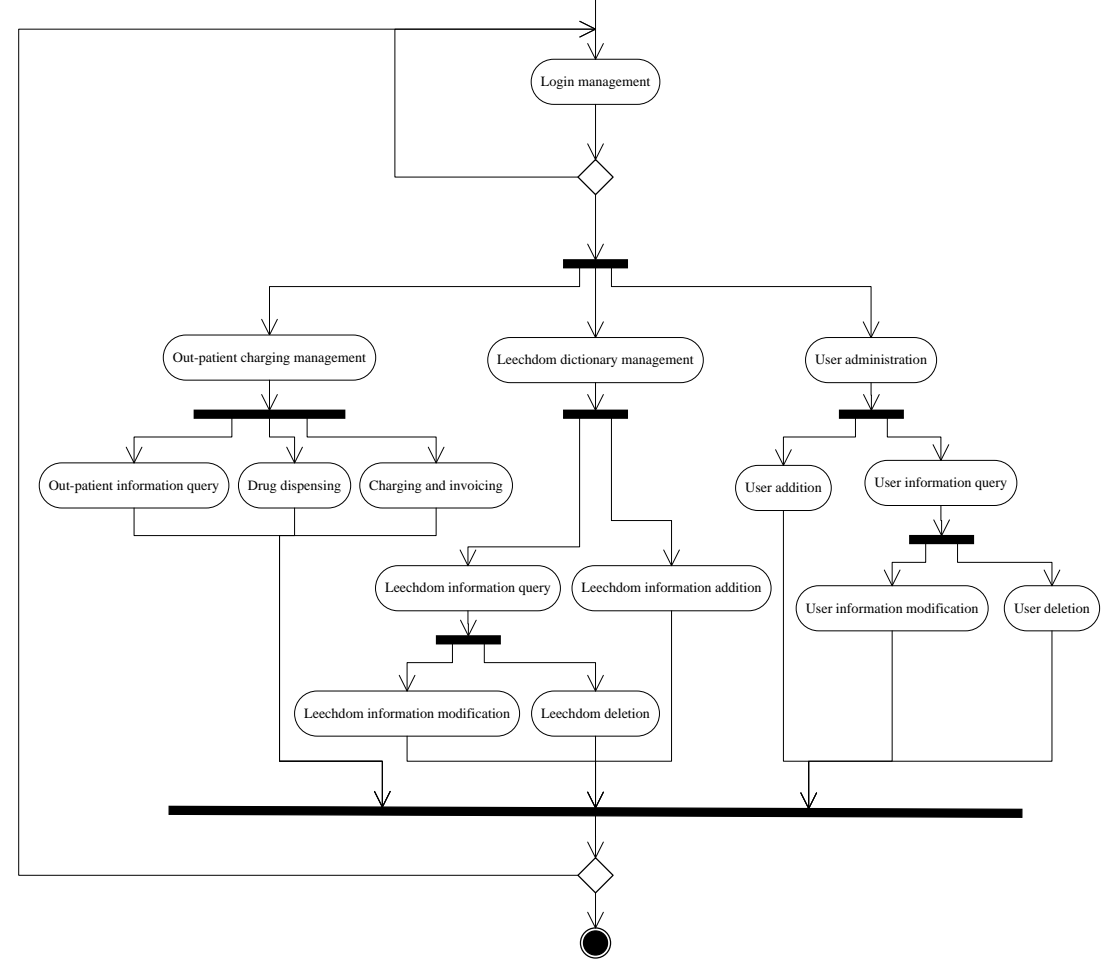

Figure 4 Activity diagram

\section{HIS sub-systems and data flows}

\section{Modules of the HIS}

According to the operation flow of the departments, the HIS is composed of several functional sub-systems. They include out-patient sub-system, medicine storehouse management sub-system, pharmacy management sub-system, electronic patient record management sub-system, leechdom dictionary management sub-system, user administration sub-system and login sub-system, as shown 
in Fig.5.

\section{Data flow}
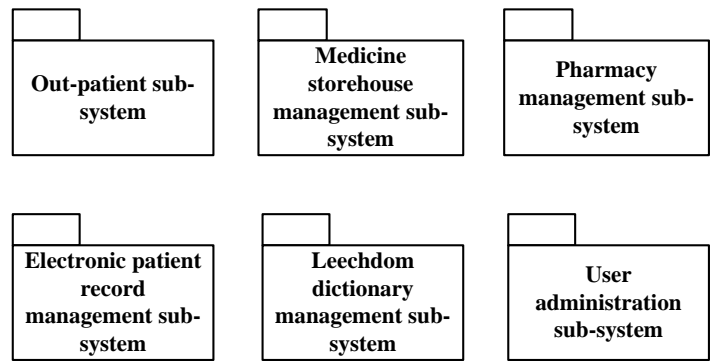

Figure 5 system modules

In the development of the HIS, the operation flows of various departments are recombined and their core flows and data flows are analyzed. Then, the operation flow of each department is determined.

The operational function of out-patient department is as follows. After a physician issues a prescription and examination list, the patient goes to the pricing and charging department to pay. Then, the patient takes the prescription and invoice to the pharmacy to get drugs or to corresponding functional departments to examine. The data flow of pricing, charging and drug dispensing is shown in Fig.6.

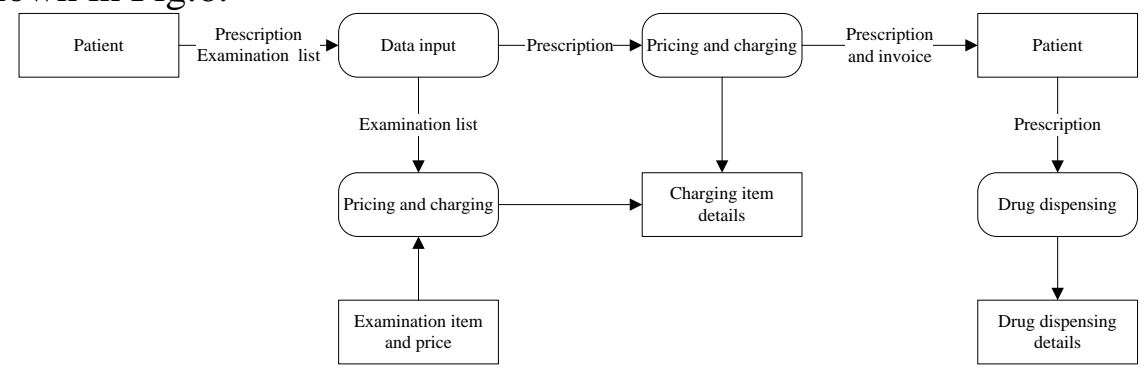

Figure 6 Data flow of pricing, charging and drug dispensing

\section{Conclusions}

In the recent years, HIS developed quickly due to the great input from government, hospitals and software companies, etc., and a lot of achievements have been obtained. But there are also many problems to be solved, such as, low application level, poor system security, low standardization level, etc. In the paper, HIS requirements are analyzed and its UML diagrams are drawn. According to the operation flow of the departments in a hospital, the HIS is dvivided into seven functional subsystems. The operation flow of various departments is recombined and their core flows and data flows are analyzed. Then, the operation flow of each department is determined. The discussion in the paper defines the system requirements and architecture for further system realization.

\section{Acknowledgements}

The paper is supported by Special Scientific Research Project of Shaanxi 13115 Scientific and Technological Innovation Engineering Project (2010FWPT-05), Shaanxi Provincial Department of Education (09JK475), Shaanxi Major Subject Construction Project and President Scientific Research Fund of Xi'an Technological University. 


\section{References}

[1] Y. Cao, L.N. Yang, H. Cao, Y. Tan, S. Cao, Design and Development of a Hospital Information System in China, Journal of Investigative Medicine 61(4S), S10-S10 (2013)

[2] Y.H. Yang, C. L. Zhu, The Effects of Hospital Information System on Hospital Management, Information of Medical Equipment 18(5), 51-53 (2003)

[3] S. B. Liu, X. X. Zhou, Analysis on Hospital Information System and Clinical Information System, Journal of Medical Informatics 30(10), 14-16 (2009)

[4] D. K. Wang, Y. L. Yu, Y. Yang, Y. H. Wang, Q. Wang, Y. Niu, X. C. Chen, 3-level-structure Hospital Information System and its Successful Application in Hospitals: the Development of Digitalized Hospitals in China, Chinese Hospitals 7(12), 23-24 (2003)

[5] X. J. Liang, Z. Sun, The application of electronic medical records system based on HL7 CDA standards and XML technology, China Digital Medicine 3(3), 21-24 (2008) 